\title{
NUEVOS MECANISMOS DE INTERVENCION EN LA CIUDAD EXISTENTE La recualificación a través del decrecimiento. Objetivo: una ciudad resiliente a través de un urbanismo circular
}

\section{Gorka Cubes San Salvador del Valle.}

ETSAM/UPM. Escuela Superior de Arquitectura de Madrid. Universidad Politécnica de Madrid. Programa de Doctorado en Sostenibilidad y Regeneración Urbana.

Director de Tesis: Jose Miguel Fernández Güell

gorkacubes@me.com

\section{RESUMEN}

El modelo expansivo imperante, no funciona cuando se enfrenta a la regeneración urbana. Mientras la ciudad siga creciendo, se limita la oportunidad para que la ciudad pueda iniciar el proceso de regeneración de una forma endógena. Es necesario un cambio sistémico. El presente artículo reflexiona sobre un nuevo modelo, que no sólo limita el crecimiento, sino que sugiere un decrecimiento programado, como método para catalizar los procesos de regeneración urbana. El decrecimiento como fenómeno en sí mismo resiliente, que de acuerdo con el paradigma de la economía circular, pueda entender el desperdicio como alimento de un nuevo proceso paralelo. Si basura es comida, el decrecimiento genera valor. Este nuevo enfoque de uso del suelo es el primer paso hacia el urbanismo circular, nuevo concepto acuñado en el presente artículo, entendido como el cierre del ciclo de vida en el uso del suelo.

Palabras clave: Decrecimiento, Resiliencia, De la cuna a la cuna, Regeneración urbana.

\section{ABSTRACT}

The current expansive model does not work when faces with urban regeneration. As long as the city continues to grow, the opportunity so that the city can initiate the process of urban regeneration in an endogenous way is limited. A systemic shift is necessary. This article reflects on a new model, which not only limits growth, but also suggests a programmed decrease, as a method to catalyze the processes of urban regeneration. Degrowth as a resilient phenomenon itself, which, according to the circular economy paradigm, can understand waste as food for a new parallel process. If garbage is food, degrowth generates value. This new approach to land use is the first step towards circular urbanism, a new concept presented in this article, understood as the closure of the life cycle in land use.

Key words: Degrowth, Resilience, Cradle to Cradle, Urban regeneration. 


\section{INTRODUCCION. CRISIS SISTÉMICA}

No parece admitir dudas el dato de que el tejido urbano y el parque edificado de nuestro entorno, próximo a alcanzar su ciclo de vida lógico, necesita intervenciones de mejora de la eficiencia y sostenibilidad; sustitución, rehabilitación, regeneración y renovación urbanas. Operaciones de adecuación a los estándares de sostenibilidad, calidad, confort y accesibilidad actuales. No es un concepto exclusivamente local, sino que se trata de una reflexión a nivel global, en el mundo occidental ${ }^{1}$. Así mismo la crisis ecológica, consecuencia directa del modelo social y económico vigente, fundamentado en la explotación de los recursos del planeta más allá de todo límite, se continúa agravando. Este modelo económico imperante, se construye sobre la absurda creencia de que el crecimiento perpetuo e ilimitado es posible. Éste el auténtico nudo gordiano que hay que erradicar ${ }^{2}$.

Se está produciendo una inversión en la pirámide de la población ${ }^{3}$ en nuestro entorno, comenzando incluso un descenso neto de población en muchos casos. Esta tendencia de envejecimiento se lleva sosteniendo en el tiempo desde hace décadas, y todos los datos apuntan a una progresión de esta tendencia por la baja natalidad y una progresiva menor llegada de población extranjera, que nos aboca a un decrecimiento en población. Las próximas generaciones, aquellas que ocupen lo que hoy urbanísticamente planifiquemos, generarán una demanda de suelo y techo mucho menor, aun teniendo en cuenta la fragmentación y disminución de las unidades habitacionales y núcleos familiares. Si a ello se une el dato del stock de vivienda vacía, llegaremos a la rápida conclusión de que la ciudad corre el riesgo de difuminar su masa crítica, dentro de un tejido urbano consolidado con múltiples carencias dotacionales y baja eficiencia. Debemos tratar de convertir esta amenaza en una oportunidad. La necesidad de sustitución o regeneración integral, concede la oportunidad de intervenir en la trama urbana existente. Debemos ser capaces de hacer conjugar esta necesidad de renovación con la regeneración urbana y la mejora de las dotaciones y equipamientos públicos. En definitiva en la mejora de la calidad del espacio y suelo urbano. Tal y como se establece en la Carta de Leipzig sobre ciudades europeas sostenibles ${ }^{4}$ es necesario configurar una nueva 'alianza urbana' 5 para poner en práctica el compromiso estratégico hacia la regeneración urbana integrada ${ }^{6}$.

El objetivo básico de las políticas de regeneración es evitar los procesos de exclusión social. Así el objetivo no es rehabilitar sino regenerar integralmente: recualificar. Desde el punto de vista operativo, la regeneración es lo contrario del urbanismo convencional: mientras el urbanismo convencional reparte el beneficio neto, la regeneración siempre comporta costes netos. El protagonista del urbanismo es, básicamente, el propietario de suelo mientras que la regeneración corresponde al usuario (aunque sea propietario) que se queda, por ello en el urbanismo el perfil técnico requerido tiene que ver con la construcción y en la regeneración con el mediador social. Se hace necesario un nuevo enfoque.

La dificultad de la regeneración urbana radica precisamente en que requiere un cambio de paradigma. Debemos enfrentar el problema sistémico que acucia al urbanismo actual: la práctica imposibilidad de intervención efectiva en la ciudad existente con el modelo actual ${ }^{7}$. Mientras la ciudad crece va dejando a un lado la regeneración de la ciudad existente porque el modelo no favorece su regeneración, porque no existen mecanismos para acometer el levantamiento de las cargas inherentes al suelo usado y obsoleto: degradación, contaminación del suelo, realojos, insolvencias, y un largo etcétera que el modelo no prevé solventar, salvo a través de un aumento artificioso del valor ${ }^{8}$ del suelo. Los instrumentos actuales no funcionan porque no existen límites y esto se evidencia en la plausible degradación urbana de la trama urbana más desfavorecida. El cambio debe ser sistémico. Las políticas de regeneración requieren la redefinición de la intervención pública demandando un nuevo estilo, menos reglamentista y más concertador. Las actuaciones de regeneración requieren modelos más abiertos, como de hecho ya intuyó tímidamente la Ley 8/2013 de Rehabilitación, Regeneración y Renovación Urbanas, y la sucesiva Ley del Suelo y Regeneración Urbana 7/2015, menos procedimentado y más dúctiles, en los que el objeto de la regulación comienzan a no ser las garantías de los propietarios, sino los objetivos, básicamente, de inclusión social.

\subsection{EL URBANISMO NO CUMLE CON SU FIN SOCIAL}

El urbanismo, en lo que se refiere a la intervención en la ciudad existente, vista tanto desde una perspectiva histórica como actual, no consigue hacer cumplir su función pública: La Función Social ${ }^{9}$, que no es otra sino el aumento de la calidad de vida, el derecho a una vivienda digna y adecuada; $y$ a un entorno urbano saludable y eficiente. La planificación urbana, como disciplina no aislada, sino interconectada e integrada con lo colectivo en su conjunto, debe acometer cambios más profundos que los exclusivamente ligados a la transformación 
física y que son especialmente problemáticos en lo que se refiere a la exclusión urbana de las periferias espaciales y sociales.

El objetivo final, debe ser cumplir con el fin social inherente a cualquier función pública, como lo es el urbanismo: mejorar la calidad de vida de las personas, globalmente entendida, no sólo susceptible de atender los objetivos parciales, de eficiencia energética y de recuperación urbana y económica ya expresados, sino también de contribuir activamente a la sostenibilidad ambiental y a la cohesión social dotando de los recursos espaciales necesarios para el desarrollo de las funciones urbanas básicas. El urbanismo visto con esta concepción de función pública con un inherente fin social, debe marcarse como objetivo acabar con las desigualdades socio-espaciales y el sistema que las genera. Vivimos de hecho en ciudades fragmentadas, debido a la diferenciación social en el de uso de espacio. Cómo reequilibrar esa distinción social-espacial, debe ser motor y objetivo de las estrategias de regeneración urbana a implementar.

Todos los enfoques y textos legales desde el año 1956, incluso la vigente constitución Española ${ }^{10}$, a pesar de que en su motivación describen la citada función social del suelo, en su transformación (nueva ciudad) y sobretodo en su conservación (ciudad existente), no le dan cumplimiento. Dicho fin social y por lo tanto el de la regeneración urbana, tiene un alcance que va más allá de la simple transformación espacial y remite a la reorganización de las relaciones sociales ${ }^{11}$.

\section{CAMBIO DE PARADIGMA: NECESIDAD DE UN NUEVO MODELO}

El urbanismo que conocemos ha de acometer un cambio de paradigma, un cambio profundo y valiente, porque no hay otra solución que se base en alteraciones al modelo actual. La ciudad se cae, la estamos dejando caer. Tenemos que intervenir o propiciar la intervención. Las soluciones a base de continuar con un modelo de ciudad expansionista no puede, ni debe continuar a base de dejar atrás un artefacto ineficiente, costoso al que no hay forma de alterar. Se debe sustituir del modelo tradicional expansivo, por el de regeneración del medio urbano actual.

Para poder empezar a acometer esta tarea tenemos que desarrollar nuevos instrumentos. Las nuevas leyes estatales en materia de regeneración y renovación urbanas, abren la puerta a definir mecanismos de intervención distintos a los del suelo urbanizable, con una mayor vocación de cumplimiento de su fin, invocando, por ejemplo, la función social del derecho de propiedad, como mecanismo para la determinación de la obligatoriedad de participar en las actuaciones de rehabilitación, regeneración y renovación urbana.

\subsection{FRENAR EL CRECIMIENTO: DECRECIMIENTO Y BUSQUEDA FORMULAS QUE CATALICEN LA REGENERACION}

Debemos partir de estos dos supuestos básicos: que el crecimiento tiene límites, y que ya los hemos sobrepasado, o estamos muy próximos a ello. Y desembocar por tanto en un corolario inmediato: debemos frenar el crecimiento. La clave está en aumentar dentro de este contexto el bienestar de las personas. Para ello la clave es la cooperación como raíz teórica, como intento de explicar el concepto de decrecimiento: el de trabajo de reciprocidad ${ }^{12}$ y las implicaciones para la formación de capital social, en un contexto de decrecimiento ${ }^{13}$. Frente al paradigma del desarrollo sostenible dominante, la hipótesis del decrecimiento sostenible ${ }^{14}$. Decrecimiento no sólo como disminución del consumo tal y como hasta ahora lo entendemos ${ }^{15}$, sino con la idea de consumo fuerte y sostenible ${ }^{16}$. Cabe interpretar este movimiento no como un factor depresivo sino catalizador hacia la verdadera función social del urbanismo.

Las diferentes variantes de las estrategias de decrecimiento ${ }^{17}{ }^{18}$ comparten la perspectiva de una mayor democratización ${ }^{19}$ además de la conciencia ambiental ${ }^{20}$. El decrecimiento se presenta de esta forma como una vía de transición hacia un futuro social y ecológicamente sostenible ${ }^{21}$. Así, el enfoque del decrecimiento se plantea más fundamentalmente como cuestiones relativas a la relación entre la prosperidad material ${ }^{22}$ y el bienestar individual y social. ${ }^{23}$. El movimiento Cohousing, por ejemplo, es un modelo para hacer la vida más social y más verde en un contexto urbano. Cohousing encaja perfectamente bien con las teorías económicas del decrecimiento ${ }^{24}$. La clave es si esto es posible sin traumáticos cambios en material institucional ${ }^{25}$. ¿Bajo qué condiciones puede ser socialmente sostenible ${ }^{26}$ Se necesita un cambio cultural y político entrelazado que abrace el decrecimiento como un desarrollo social positivo y la reforma de las instituciones. Por lo tanto, el decrecimiento sostenible no es sólo un concepto estructurante; es un proyecto político y social que ofrece un nuevo lema de agrupamiento para una coalición social construida alrededor de la aspiración de construir una sociedad que vive mejor con menos ${ }^{27} 28$. 


\subsection{LA CIUDAD COMPACTA Y DENSA}

En la actualidad, existe una investigación continuada acerca de la densidad urbana. En general, se puede afirmar que hay una tendencia a la preferencia por ciudades de media-alta densidad que combinen de forma correcta eficiencia con calidad de vida. No obstante, se reconoce que, ya sea por los costes en materia de congestión o ruido de la ciudad compacta, o por el masivo desplazamiento en coche privado que conlleva la ciudad difusa, en ambos casos existen problemas, porque la ciudad ideal, simplemente, no existe, y además no existe una solución técnica al problema ${ }^{29}$. En cualquier caso, existen algunos intentos por llegar a la formulación óptima ${ }^{30}$ de relación entre el suelo y techo; y todos ellos coinciden en el concepto de redensificación.

La ciudad compacta se asocia, en muchos casos y de forma simplista y vaga, a ciudad vertical, a ciudad construida con edificios altos. Sin embargo, la asociación más correcta y necesaria en la concepción de esta clase de ciudad es la de compacidad y densidad, términos con una referencia clara a un desarrollo urbano continuo y denso. El concepto de ciudad compacta no sólo se refiere a la forma física de la misma, sino que implica una compacidad de funciones, una mezcla e interrelación de actividades, favorecidas por la densidad, que comparten un mismo tejido urbano. Las características esenciales en las ciudades compactas son: contacto, regulación, intercambio y comunicación ${ }^{31}$. La ciudad compacta y densa es aquella que aumenta la complejidad de las partes del sistema de ciudad generando una vida social cohesionada y una plataforma económica competitiva. La mezcla, la hibridación, la proximidad de la vivienda, el comercio, el teatro, el parque, el colegio... son componentes inseparables de la ciudad compacta y una de sus cualidades más positivas y garantía de una austeridad en el uso de las energías necesarias para su funcionamiento.

\subsection{LA RESILIENCIA}

¿Qué tiene que ver la resiliencia con la cuestión urbana? ${ }^{32}$ El concepto de resiliencia urbana es la capacidad de una ciudad expuesta a una amenaza para resistir, absorber, adaptarse y recuperarse de sus efectos de manera oportuna y eficiente, lo que incluye la preservación y restauración de sus estructuras y funciones básicas. Esta resiliencia está vinculada a los conceptos dinámicos de desarrollo urbano ${ }^{33}$. En este sentido, la resiliencia es un proceso y no una respuesta inmediata a la adversidad ${ }^{34}$. Ser resiliente tiene poco, por no decir nada, que ver con ser invulnerables, sino con el aprendizaje y la adaptación ${ }^{35}$. La resiliencia urbana no es una nueva técnica de gestión de emergencias, es alguna cosa más. Es más bien una invitación a tener una nueva mirada sobre el desarrollo de la ciudad.

La cuestión crítica es lo que las ciudades y sus comunidades urbanas pueden y deben hacer para avanzar hacia un estado más resiliente en el futuro ${ }^{36}$. El concepto de "gobierno urbano", la gobernanza, contribuye a la gestión integral de la resiliencia urbana. Existe una necesidad significativa de un nuevo enfoque hacia la gobernanza urbana para hacer frente a las incertidumbres ya los futuros desafíos ambientales y de cambio climático ${ }^{37}$.

\subsection{CRADDLE TO CRADDLE ${ }^{38}$}

Tradicionalmente la consigna principal del ecologismo ha sido Reducir, reutilizar, reciclar. Esta nueva filosofía propone un cambio de enfoque. Reducir el impacto sobre el medioambiente provocaría una ralentización del mismo, pero más rápido o más despacio estaríamos llegando a un mismo final. Frente a este panorama se propone que se atajen los problemas desde su misma raíz, es decir, que en vez de reducir los consumos de energía, nos centremos en que desde el propio diseño y concepción de cualquier producto, estrategia o política se tengan en cuenta todas las fases de los productos involucrados (extracción, procesamiento, utilización, reutilización, reciclaje...) de manera que ni siquiera sean necesarios los gastos de energía, incluso que el balance de gastos y aportes sea positivo.

Los conceptos clave de la filosofía "de la cuna a la cuna" son intuitivos y enraizados en la imitación a la naturaleza. El cierre completo de los ciclos de materiales: en los ecosistemas del planeta, no existe la basura. La basura es el alimento del siguiente ciclo. Nuestras sociedades pueden hacer lo mismo diseñando todos los productos de modo que los materiales se reciclen en el mismo uso, o bien se reciclen "hacia arriba", es decir que el siguiente uso tenga más valor que el actual. 


\subsection{LA ECONOMIA CIRCULAR ${ }^{39}$}

Derivado de la filosofía cradle to cradle surge el concepto más global de economía circular. La economía circular es un concepto económico que se interrelaciona con la sostenibilidad, y cuyo objetivo es que el valor de los productos, los materiales y los recursos (agua, energía,...) se mantenga en la economía durante el mayor tiempo posible, y que se reduzca al mínimo la generación de residuos. Esta es su principal diferencia con el cradle to cradle puesto que éste sin embargo celebra la abundancia. En este caso, se trata de implementar una nueva economía, circular -no lineal-, basada en el principio de "cerrar el ciclo de vida" de los productos, los servicios, los residuos, los materiales, el agua y la energía. La economía circular es la intersección de los aspectos ambientales y económicos. Por lo tanto, la economía circular propone un nuevo modelo de sociedad que utiliza y optimiza los stocks y los flujos de materiales, energía y residuos y su objetivo es la eficiencia del uso de los recursos.

\section{NUEVO MECANISMO: IF "GARBAGE IS FOOD"... HIPOTESIS: "DEGROWTH GENERATES VALUE"}

Tomando el diagnóstico efectuado y el análisis de las ideas, estándares y tendencias de estado del arte señaladas, surge el siguiente presupuesto conceptual: Atendiendo al paradigma de la eficiencia a través de la densidad, la redensificación urbana debe pasar ineludiblemente por la desocupación de parte de suelo colmatado. No es materialmente posible alcanzar la eficiencia aumentando la densidad urbana, sin conseguir en paralelo la desocupación de lo no denso, o no consolidado, de lo contrario se haría crecer el techo edificado neto del conjunto del sistema urbano, consiguiendo el efecto contrario de pérdida de eficiencia (paradoja de Jevons).

Las operaciones urbanísticas tradicionales ponderan el valor del suelo mínimo para que la operación de promoción o renovación urbana sea factible y cubra al menos el valor de reposición de la edificación existente en el momento inicial; añadidos los gastos de la transformación y las cesiones urbanísticas legalmente establecidas. La realidad nos indica que cualquier operación de regeneración urbana, para conseguir movilizar el suelo urbano consolidado, debe programar un aumento de valor: un aumento de su edificabilidad urbanística, una expectativa, para poder ser rentable en las condiciones de partida, para poder llevarse a cabo y asumir los costos inherentes al desmontaje del artefacto existente. Siempre y cuando, no se recurra al intervencionismo público, el cual no sólo debe evitarse o no establecerse como método a seguir, si no que va a ser cada vez menos recurrente, sostenible y justificable.

Dado que se hace necesario un aporte de valor adicional al establecido para conseguir la movilización pretendida. Descartado el aumento de la edificabilidad, ni de la subvención pública a fondo perdido; teniendo en cuenta la dispersión y pérdida efectiva de la masa crítica urbana de la periferia... surge un nuevo mecanismo de intervención y vía de aportación del incremento de valor necesario, de forma indirecta: ¿Y si ese valor adicional, ese incremento, o cuestión de oportunidad, viniese dado de una forma exógena al propio ámbito., pero que finalmente catalice la regeneración de forma endógena? Surge, una respuesta diferente, un nuevo mecanismo de intervención en la ciudad existente: el decrecimiento como generador de valor. Decrecimiento activo y programado como medio de concentración de la demanda, mediante la restricción de la oferta y catalizador de la regeneración urbana.

Las curvas de la oferta y la demanda en su progresión son logarítmicas, no lineales. El incremento de valor es exponencial conforme la oferta disminuye. La formulación logarítmica es inversamente potencial, la cuestión clave residirá en el equilibro que consiga satisfacer la demanda y cuadrar los costos. El reto es articular un nuevo modelo, regular y procedimentar mecanismos de decrecimiento como medio para recualificar la trama urbana: aumentar la eficiencia, la eficacia, la sostenibilidad, reducir la huella ecológica y aumentar el valor de la trama, y por ende la calidad de vida de los ciudadanos. Fórmulas que primen el aumento de valor y de cualificación urbana a través de fórmulas de desocupación de suelo y de techo construido en algunas zonas, en paralelo con la recualificación y el esfuerzo integrado en otras ${ }^{40}$. 


\section{NUEVA APORTACION: UNA CIUDAD RESILIENTE A TRAVES DE UN URBANISMO CIRCULAR. (URBANISMO CIRCULAR ENTENDIDO COMO EL CIERRE DEL CICLO DE VIDA EN EL USO DEL SUELO)}

El concepto último que pretende catalizar el mecanismo mencionado es el Urbanismo Circular. Este nuevo concepto que avanza el presente artículo, trata de la incorporación de los conceptos de economía y producción circular al urbanismo y al uso del suelo, con el objetivo de crear ciudades inteligentes, que sean capaces de adaptarse y aprender de sus propios errores: ciudades resilientes. Definitivamente en el planeamiento se debe contemplar el fin de vida de las edificaciones, la vinculación del uso efectivo a la calificación y clasificación del suelo, el cierre del ciclo de vida del uso del suelo y huir de los modelos lineales ${ }^{41}$. Para poder alcanzarlo, el suelo se debe poder ocupar y desocupar con la misma facilidad.

El objetivo último de la ciudad resiliente sólo se puede alcanzar, aceptando el nuevo paradigma de la producción y la economía circular. Como apuntan los autores Michael Braungart y William McDonough en "cradle to cradle" el cambio de paradigma pasa por la idea de que en cualquier proceso productivo, la basura se debe entender como alimento de un nuevo proceso paralelo. A imagen y semejanza de los procesos naturales... Ya no es únicamente necesario pensar en los conceptos tradicionales del ecodiseño en términos de reducción de la producción de residuos y mínimo consumo de energía. Se puede y debe celebrar la abundancia y la diversidad siempre que el proceso sea cerrado y que el residuo de un proceso sirva o bien como alimento biológico de dicho proceso, o bien como alimento tecnológico de un proceso paralelo, pero siempre en un esquema circular, de retroalimentación totalmente eficiente ${ }^{42}$.

El urbanismo circular: El cierre del ciclo de vida en el uso del suelo. Para poder alcanzarlo, como se ha dicho anteriormente, el suelo se debe poder ocupar y desocupar con la misma facilidad. Esta flexibilidad en el uso del suelo es el primer paso hacia la resiliencia y el urbanismo circular. La resiliencia está vinculada a los conceptos dinámicos de desarrollo y de transformación urbana, y nunca se podrá alcanzar plenamente si no se aplican mecanismos que faciliten el decrecimiento. El decrecimiento es en sí mismo resiliente ${ }^{43}$ : La resiliencia es un proceso y no una respuesta inmediata a la adversidad. Como reacción a la agresión, se debe contemplar la respuesta de la retracción como la más resiliente y posibilista de todas.

El urbanismo de un futuro viable debe ser el circular. Transformación y transición, dos conceptos que deben orientar la redacción de planeamiento en el futuro inmediato. Transformación implica en primer lugar abandono de la expansión, pero no sólo eso: comporta que la intervención sobre la ciudad existente persiga la reducción de los consumos, haciéndola mismo tiempo más amable, más habitable. Deberá centrarse en la mejora de lo existente, del stock acumulado de suelo y vivienda, en gran medida vacante; en la recualificación de las áreas urbanas consolidadas, en el incremento de las dotaciones, de los espacios verdes; en la reducción de la demanda de movilidad motorizada, uno de los factores de mayor deterioro del medio urbano. Por decirlo resumidamente: en la reducción de insumos de los consumos, especialmente de los no renovables, pero también de los renovables, para acercarlos a su tasa de reposición. Este es el objetivo estratégico para la consecución de un urbanismo circular: la reducción tanto del consumo de materias primas como de la generación de residuos, partiendo de la premisa del no crecimiento.

Una vez conseguido un mejor funcionamiento del artefacto (arquitectura, territorio o ciudad) queda una segunda parte tan importante como la primera: cómo conseguir que estas mejoras en el funcionamiento repercutan en el reequilibrio entre territorios o clases sociales, en definitiva al cumplimiento del fin social del urbanismo. Estaremos más cerca de lograrlo sin duda si contamos con un modelo más eficiente, sostenible y flexible.

\section{FACTIBILIDAD. CATALIZADORES}

A continuación se identifican de forma no exhaustiva, los elementos que impiden que este axioma se cumpla, con especial incidencia en aquellos de éstos que con su transformación pueden servir de catalizadores en la dirección pretendida.

\subsection{SOCIOLOGIA INDETITARIA. HACIA EL NO LUGAR COMO ESPACIO DE HABITACIÓN}

En primer lugar, uno de los elementos clave sociológicos a aplicar y que puede ayudar a catalizar el proceso, es sin duda la transformación y rotura de la vinculación identitaria del ciudadano con su lugar de procedencia físico y su reticencia a la movilidad. No sólo el lugar de origen como ciudad o región sino así mismo la 
vinculación sentimental con su propia vivienda. Este es un concepto ancestral que lastra la transformación urbana, por el atávico terror y reticencia a abandonar el propio hogar y cambiarlo por otro, aunque sea este último mucho mejor. Se debe atacar la falta de movilidad y el desapego al refugio como vivienda que data del imaginario prehistórico.

A modo de ejemplo, el mundo del trabajo y las relaciones entre sus protagonistas ha cambiado de una manera asombrosa en los últimos años, y lo seguirá haciendo exponencialmente en los siguientes. Las nuevas condiciones económicas que afectan la producción y sus relaciones laborales llevan a una tendencia fuerte hacia la fragilidad del vínculo entre los empleados y las empresas. El tradicional concepto de "relación de dependencia" es cada vez más efímero, más breve, y se tiende al vínculo jornalizado, sobre el antiguo concepto de asalariado. Esta misma desvinculación se debe buscar entre el habitante y la vivienda, se debe dar paso a un concepto más utilitario. Al respecto merece la pena analizar lo que alguno autores denominan y proponen la hipótesis que son "no lugares" como concepto útil a proponer como condición de contorno para poner en práctica la idea planteada. Se debería comenzar a hablar también de una nolugarización en relación con el espacio residencial. La planificación que se lleva a cabo en las ciudades y en la arquitectura de nuestro entorno no acaba de asumir el tiempo pasado y de proponer el alcance de lo que debe ser una nueva forma de concebir los espacios, su funcionalidad y el espacio de relación y comunicación, con el objetivo de conseguir una menor vinculación del habitante con el lugar habitado que facilite su movilidad y limite su reticencia a cambiar sus hábitos y patrones en cuanto a los espacios de vivienda y relación.

\subsection{TEMPORALIDAD DE LA CALIFICACION Y CLASIFICACION}

Se debe ligar el espacio y el tiempo, es decir: la ocupación, el uso del suelo y su caducidad. Se debe ligar con mayor fuerza la clasificación y calificación fijando su valor con la vigencia de los planes en su ámbito temporal y no más allá. Los planes califican y clasifican el suelo, pero únicamente en lo que estos sean vigentes, aunque parecemos olvidarlo. En el suelo urbanizable no desarrollado, si no se cumplen los plazos de ejecución, también debe perder esa condición. La dimensión temporal no se aplica con rigor y debe ser indisoluble. Este es el primer paso hacia la resiliencia y el urbanismo circular entendido como cierre del ciclo de vida del uso del suelo.

Este concepto es crítico aplicarlo, así como el fin de vida del suelo artificializado, para poder realmente aplicar el concepto de urbanismo circular. El planeamiento debe incidir en otorgar usos e intensidades de forma limitada en el tiempo para favorecer una construcción menos pétrea y una conciencia de fin de vida del edificio y del uso de suelo. Es crítico, como ya se ha comentado antes, que se comience a cumplir de forma verdadera y efectiva, lo que ya de por sí hoy en día es normativo, pero no se respeta: la calificación y clasificación del suelo no es eterna: lo es en tanto en cuanto el plan tiene vigencia y se puede y debe renovar con similar periodicidad.

Por otra parte, la programación de los planes, en lo que se establecen la cronología de desarrollo de cada uno de los sectores, en el caso de no cumplirse debería dar origen a la inmediata revisión del plan, previo paso inicial de la desclasificación y descalificación de dicho suelo. Esta idea permitiría catalizar los procesos de regeneración de forma efectiva porque los plazos si no se cumplen se pierden la clasificación.

\subsection{DEBER DE CONSERVACIÓN. NUEVOS MECANISMOS DE FINANCIACION}

La ley prevé, que todos y cada uno de los propietarios de suelo y techo, tenemos el deber de conservarlo en perfectas condiciones de seguridad, salubridad y ornato público, pero nos encontramos con una directriz que no se cumple. Se debe cumplir este precepto y no esperando a las subvenciones. Las subvenciones a la rehabilitación producen una sensación de eficacia a corto porque se traducen en intervenciones pero tenemos que asumir que no hay, ni va a haber, dinero público para subvencionar las actuaciones de regeneración, ni de rehabilitación. De hecho los instrumentos europeos ya apuntan en ese sentido, hacia un cambio ${ }^{44}$. Necesitamos fomentar una nueva cultura urbana y de la conservación basada en la responsabilidad de los propietarios eliminando las políticas de ayudas, porque deseducan. Una nueva política "tributaria" que refleje el verdadero coste de los servicios y dotaciones públicas, incluyendo su mantenimiento, conservación y amortización para garantizar su sostenibilidad. Pero si no cambiamos la expectativa de las subvenciones por mecanismos más justos y con capacidad de replicabilidad, nos dirigimos directamente hacia la inacción sobre el medio urbano, bloqueo de la regeneración que no nos podemos permitir. Por ello, hemos de dejar de circular en dirección contraria, ponernos a dar pasos hacia nuevos mecanismos de financiación, que no de subvención, para desencallar las actuaciones de regeneración. 
Así mismo, el deber de conservación que por ley obliga al propietario está sujeto a unos límites que se deben superar, ${ }^{45}$ que se concretan en la situación de ruina, y más concretamente en la ruina económica, donde, por una parte, no es exigible al propietario la conservación, dando paso a la demolición, (que debería dar paso a o bien la recuperación del bien por parte de la comunidad, o bien la desclasificación del suelo) y, por otra parte, surge el deber de la Administración de costear las obras en tanto se exceda del deber legal de conservación con el correlativo derecho del propietario a la indemnización. Esto no puede seguir así.

Los nuevos instrumentos financieros deben buscar ineludiblemente la conciliación de las inversiones públicas y privadas, redefinir el papel de la participación en las plusvalías a corto y a largo; salvar los ámbitos temporales clásicos de retorno de las inversiones a través de una metodología holística, redefinir las valoraciones contables de suelo, vuelo y uso; y modificar los conceptos de bienes muebles e inmuebles junto con la transversalidad entre áreas de actuación.

\subsection{FACULTAD DE PARTICIPACION. TRATAMIENTO DE LAS INSOLVENCIAS}

Aun así, siempre hay propietarios que no pueden "pagar". Esta circunstancia ha sido una de las cuestiones por la cual en muchas ocasiones no se han activado las actuaciones de regeneración y a renglón seguido se ha venido sosteniendo como un mantra que la solución son las ayudas y subvenciones públicas, esto es, entregar el dinero de todos, gratis. Pero esto es insostenible. Las insolvencias requieren apoyo colectivo (bien a través de instrumentos financieros de corte público o mixto o como carga de la actuación de regeneración). En cualquier caso, es injustificable la aportación a fondo perdido. El apoyo es necesario pero la única opción razonable es la financiación con inscripción de las deudas como cargas a levantar a la siguiente transmisión, para convertir el problema de los impagos e insolvencias en un problema de capacidad de pago de unas cuotas más pequeñas y en cualquier caso que el instrumento financiero recupere el dinero para garantizar el efecto revolving (la reutilización de la capacidad financiera).

\subsection{VINCULACION DE LA CALIFICACION AL USO REAL}

Uno de los conceptos catalizadores claves, es la necesaria vinculación efectiva de la calificación y clasificación del suelo a su ejercicio real y efectivo. Si asumimos que el urbanismo es la potestad delegada por todos, de disponer en determinados lugares unos usos e intensidades determinados; deberemos convenir igualmente que si dicho ejercicio no se produce, es porque o bien la previsión no fue bien analizada o porque dicha previsión ya no es vigente. Con lo cual, el principio básico debería ser, que si en un suelo o vuelo no se está ejerciendo la actividad para la cual fue calificada o clasificada, dicha previsión debería decaer y perder su vigencia. La situación actual se debería superar, estableciendo la caducidad del derecho de propiedad fijado en el uso efectivo del bien calificado. Es crítico, en aras a caminar hacia el paradigma del urbanismo circular, vincular indisolublemente la calificación de suelo a un ámbito temporal y al ejercicio de su uso efectivo.

De igual modo, cuando un Plan determinado, prevé en un suelo virgen el desarrollo de una serie de usos, determinaciones e intensidades para un suelo, dicha aprobación debería (que ya lo está, pero no se tiene en cuenta) estar condicionada al desarrollo real de lo que en dicho plan se establezca. Tanto en el suelo ocupado, como en el que se prevea ocupar, si dicho uso en el primer caso, o dicha ocupación y uso en el segundo no se produce, la capacidad legal que tiene de ocuparse debería decaer.

\subsection{POTENCIACION DE NUEVAS FORMAS DE PROPIEDAD Y USO}

Otro de los elementos primarios a alterar y que pueden contribuir como catalizador hacia el urbanismo circular, es la modificación el derecho a la propiedad según hoy en día lo conocemos. De otro modo es imposible superar la petrificación urbana. Hoy se comienzan a dar pasos en ese sentido y se abre una gran variedad de nuevas formas de propiedad, comunitaria, de uso y colectiva a explorar y que otorgan elementos a valorizar que indican un nuevo camino a seguir. De la mano con la mixtificación de usos, y uniendo las fórmulas colaborativas íntimamente ligadas al uso, con la flexibilidad y adaptación prospectiva a la disponibilidad, se puede remover la petrificación del suelo y vuelo construido. Dentro de este concepto se pueden distinguir diferentes variantes de posesión con su respetiva vinculación al uso y a los derechos, pero siempre con una estrecha y directa vinculación entre ambos conceptos: derecho de propiedad y efectivo ejercicio del uso indisolublemente ligados. 


\subsection{CONSTRUCCION SOSTENIBLE: EFIMERA Y REUTILIZABLE}

Otro de los elementos sobre los que incidir y plantear alternativas catalizadora para poder caminar hacia el urbanismo circular, se centra en el tipo de construcción pétrea e insostenible al que nos enfrentamos ${ }^{46}$. Es imposible el llegar a ningún tipo de planteamiento del urbanismo circular sin poder alterar la forma de construir que tenemos. Para poder alcanzar por completo el fin de vida del uso del suelo se debe facilitar la ocupación del mismo a través de una implantación mucho más flexible. La construcción debe ser además de sostenible y además desmontable no ya sólo por motivo de la aplicabilidad de este concepto, sino desde el punto de vista del mantenimiento y conservación del parque edificado.

Los marcos legislativo, financiero y operativo de hoy no tienen la capacidad suficiente para lograr la transformación de los edificios y no podrán ofrecer las oportunidades económicas, sociales y ambientales que en este sentido se deben presentar. La nueva edificación y rehabilitación debe caminar hacia una construcción mucho menos pétrea, dentro de los conceptos de temporalidad en la calificación y uso efectivo apuntados, tendentes a una mayor versatilidad y cerrar los ciclos de vida técnicos apuntados por el concepto "de la cuna a la cuna". Esto debería ser así, tanto en la obra de edificación, como en la de urbanización y ocupación de suelo.

\subsection{VALORIZACION RCDS: RESIDUOS DE DEMOLICION Y CONSTRUCCION.}

Dentro de este concepto de modos de construcción flexible, dentro de este concepto y como elemento catalizador se debe considerar la revalorización de los RCDs (Residuos de Demolición y Construcción) dentro del proceso de regeneración de la ciudad. Como se ha apuntado, dentro de la filosofía "de la cuna a la cuna" la basura debe ser considerada como un nuevo alimento del sistema. Por lo tanto dentro del concepto de urbanismo circular, los detritus de los edificios que entren en su final de ciclo de vida, deberían ser alimento del sistema en el final del ciclo del uso del suelo. Esto lógicamente podría ser así, dentro de una concepción de la construcción que haya tenido en cuenta el final de ciclo de vida desde el propio diseño, pero hasta que este cambio de paradigma en la construcción no opere, debe existir una política pública de apoyo que prime la reutilización de estos materiales, aun con una menor eficacia que los de primer ciclo extractivo, para poder otorgar un valor adicional que haga más rentable el desmantelamiento y contribuya al cierre del ciclo de vida del uso del suelo y el urbanismo circular.

\section{CONCLUSIONES. CONSECUENCIAS}

Resumen del presupuesto conceptual: Para que haya intervención en la ciudad tiene que haber plusvalía, y mientras ésta siga creciendo y consumiendo suelo, no habrá posibilidades de generar plusvalía en la ciudad existente, por tanto hay que intervenir en la oferta limitando la expansión para, así, generar plusvalía en el suelo urbano consolidado degradado la cual catalice su regeneración. Decrecer, no como una necesidad higiénica o únicamente de reducción de la huella ecológica, sino como método para generar un aumento de valor, que haga surgir la oportunidad de la regeneración del tejido urbano existente. Una forma de concentración de valor, a través del decrecimiento neto físico, pero crecimiento neto en términos de valor resultante final medido de forma holística.

El fin es demostrar que se puede alcanzar la resiliencia y el urbanismo circular, mediante la recualificación a través del decrecimiento, en la ciudad existente. El fin último debe ser la recualificación para dar cumplimiento efectivo del fin social asociado al urbanismo.

El reto por lo tanto es contemplar el decrecimiento, no como una necesidad higiénica o únicamente de reducción de la huella ecológica, no como una actuación de mero gasto colateral al sistema, sino como método para generar un aumento de valor, que haga surgir la oportunidad en la regeneración del tejido urbano existente. Una forma de concentración de valor, a través del decrecimiento neto físico, pero crecimiento neto en términos de valor resultante final.

El objetivo por lo tanto, debe ser la planificación desde el paradigma del urbanismo circular, aquel que contempla todo el ciclo de vida en el uso del suelo y prevé desocuparlo con la misma facilidad que ocuparlo. No sólo con el objetivo de poder gestionar de una forma más racional los escasos recursos de suelo, sino porque la flexibilidad en sus uso, como hemos visto, pueden generar sinergias que permitan regenerar el suelo consolidado. Por lo tanto, se deben articular nuevas formas de proceder en este sentido, al mismo tiempo que hacer partícipe a la ciudadanía de sus obligaciones y responsabilidades, no solo de sus derechos. 
Para ello son necesarios nuevos modelos de planeamiento estratégico en vez de planeamiento encorsetado y de definición milimétrica que limitan más que habilitan.

Como conclusión del presente artículo trataré de avanzar cuales entiendo que deben ser la claves y la mentalidad, que debe guiar la planificación en el futuro, para poder conseguir el objetivo del urbanismo circular pretendido. Dos ideas o conceptos con sus procedimientos y metodologías claves se deberán aplicar:

a) Planeamiento prospectivo y directivo.

b) Agile Planning. Planeamiento continúo.

\subsection{PLANEAMIENTO PROSPECTIVO Y DIRECTIVO}

Se define como planeamiento prospectivo, aquel que pretende ligar indisolublemente y unir la oportunidad y beneficios vinculando la planificación territorial y la previsión, por adelantado, de las situaciones que podrían derivarse de sus influencias conjugadas.

El planeamiento directivo sería a su vez, aquel que no pretende una relación directa entre las necesidades y la relación y disposición espacial. El hecho de otorgar unas intensidades y usos concretos a cada porción del territorio, limita de hecho su desarrollo a esa concepción. Es necesario que el planeamiento establezca los objetivos, pero sin limitar su desarrollo espacial, de tal forma que a través de esa flexibilidad pueda implementarse de forma efectiva con una mayor efectividad las determinaciones de los fines de la ordenación. La legislación urbanística no tiene obligación de garantizar la actividad de los especuladores de suelo, al contrario, tiene la obligación de evitarla (art. 47 CE). Por lo que nada obliga a atribuir en detalle la edificabilidad a los terrenos. Podría (y en mi opinión, debería) pasarse a un modelo de planeamiento por objetivos en que el acento esté en los objetivos perseguidos para la ciudad sin fosilizar los medios. Es necesario superar el culto al plan, que es solamente un medio, para trabajar la planificación. Esto permitiría evitar que los medios sustituyan a los fines, adaptar los medios a las circunstancias de cada momento.

Ambos conceptos permitirían evitar que los medios sustituyan a los fines, adaptar los medios a las circunstancias de cada momento y eludir consecuencias indeseables. También permitiría simplificar la elaboración del planeamiento y acortar los procesos de su elaboración con lo que se reduciría el riesgo de obsolescencia y se facilitaría la rectificación de los errores.

\subsection{PLANEAMIENTO CONTINUO. AGILE PLANNING}

Ser Agile, se podría definir como la definición del planeamiento en continuo. Se Agile es tiene como principal característica realizar entregas rápidas y continuas, basadas en la multidisciplinariedad. Es decir, el proyecto se "trocea" en pequeñas partes que tienen que completarse y entregarse a corto plazo. Se trata, poner el acento en los objetivos, de poder reconsiderarlos con regularidad y alta frecuencia. Las necesidades sociales evolucionan rápidamente y experimentan cambios impredecibles hace muy poco tiempo y son elementos que condicionan drásticamente la configuración social. El drama de los desahucios, la reducción demográfica, la inestabilidad del sector energético... son elementos que condicionan drásticamente la configuración social. La capacidad de adaptación de la actividad urbanística a los nuevos problemas es nula. No se trata de poner patas arriba el planeamiento cada cuatro meses, se trata de perfilar las partes variables y articular instrumentos que permitan la flexibilidad, para que el conjunto sirva mejor a la calidad de vida de las personas. 


\section{BIBLIOGRAFIA}

${ }^{1}$ HOW IS IT THAT CITIES CAN SHRINK. Eduard Führ Cottbus. PUBLIC SPACE IN THE TIME OF SHRINKAGE. CONTENTS VOL. 8, NO. 1 (2003)

${ }^{2}$ L. Krier. "Growth: maturity or over-development?" in prashad, deependra (ed.) [2010]. New architecture and urbanism: development of indian traditions. Cambridge scholars publishing, Newcastle.

${ }^{3}$ http://wdi.worldbank.org/table/2.1

${ }^{4}$ Reunión informal de ministros de desarrollo urbano de la Union Europea. (declaración 2007).

${ }^{5}$ Carta mundial por el Derecho a la Ciudad: http://www.ugr.es/ revpaz/documentacion/rpc n5 2012 doc1.pdf

${ }^{6}$ Unión europea. Reunión informal de ministros de desarrollo urbano. Declaración de Toledo. (2010).

${ }^{7} \mathrm{M}$. Auken. Informe sobre el impacto de la urbanización extensiva en españa en los derechos individuales de los ciudadanos europeos, el medio ambiente y la aplicación del derecho comunitario, con fundamento en determinadas peticiones recibidas (2008/2248(ini)) UE. Comisión de peticiones (2009)

${ }^{8}$ UNDERSTANDING VALUE CHANGES IN THE URBAN DEVELOPMENT PROCESS AND THE IMPACT OF MUNICIPAL PLANNING Finn Kjær Christensen. Aalborg University, Department of Development and Planning, Denmark. LAND USE POLICY. VOLUME 36, JANUARY 2014, PAGES 113-121. ELSEVIER.

${ }^{9}$ N Smith, R. Rolnik, A. Ross, M. Davis. Después del neoliberalismo: ciudades y caos sistémico. Macba, (2009). Observatorio metropolitano.

${ }^{10}$ Artículo 47, Constitución Española.

${ }^{11}$ E. García. El cambio social más allá de los límites al crecimiento: un nuevo referente para el realismo en la sociología ecológica. Aposta. No. 27. (2006)

${ }_{12}$ V. Andreoni, S. Galmarini. How to increase well-being in a context of degrowth. Futures 55 (2014). Elsevier

${ }^{13} \mathrm{~V}$. Andreoni, S. Galmarini. On the increase of social capital in degrowth economy. Procedia - Social and Behavioral Sciences 72 (2013). Elsevier

${ }^{14}$ J. Martínez-Alier, U. Pascual, F.D. Vivien, E Zaccai. Sustainable de-growth: mapping the context, criticisms and future prospects of an emergent paradigm. Ecological economics 69 (2010). Elsevier

${ }^{15}$ D. W. O'neill. Measuring progress in the degrowth transition to a steady state economy. Ecological economics 84 (2012). Elsevier

${ }^{16} \mathrm{~S}$. Lorek, D. Fuchs. Strong sustainable consumption governance e precondition for a degrowth path?. Journal of Cleaner Production 38 (2013). Elsevier

${ }^{17} \mathrm{E}$. Garcia. Degrowth, the past, the future, and the human nature. Futures 44 (2012). Elsevier

$18 \mathrm{~J}$. Van den Bergh. Environment versus growth - a criticism of "degrowth" and a plea for "a-growth. Ecological economics 70 (2011). Elsevier

${ }^{19}$ M. Deriu. Democracies with a future: degrowth and the democratic tradition. Futures 44 (2012). Elsevier

${ }^{20} \mathrm{~K}$. Ott. Variants of de-growth and deliberative democracy: a habermasian proposal. Futures 44 (2012). Elsevier

${ }^{21}$ N. Videira, F. O. Shneider, F. Sekulova, G. Kallis. Improving understanding on degrowth pathways: an exploratory study using collaborative causal models. Futures 55 (2014). Elsevier

22 Índice de bienestar económico sostenible (IBES). Http:/lecosdelfuturo.com/glosario/indice-de-bienestar-economico-sostenible/

${ }^{23} \mathrm{C}$. Bauhardt. Solutions to the crisis? The green newdeal, degrowth, and the solidarity economy: alternatives to the capitalist growth economy from an ecofeminist economics perspective. Ecological economics 102 (2014). Elsevier

${ }^{24} \mathrm{M}$. Lietaert. Cohousing's relevance to degrowth theories. Journal of cleaner production 18 (2010). Elsevier

${ }^{25} \mathrm{~F}$. Schneider, G. Kallis, J. Martinez-Alier. Crisis or opportunity? Economic degrowth for social equity and ecological sustainability. Introduction to this special issue. Journal of cleaner production 18 (2010). Elsevier

${ }^{26} \mathrm{G}$. Kallis, C. Kerschner, J. Martinez-Alier. The economics of degrowth. Ecological economics 84 (2012). Elsevier

${ }^{27} \mathrm{G}$. Kallis. In defence of degrowth. Ecological economics 70 (2011). Elsevier

${ }^{28}$ A. Matthey. Less is more: the influence of aspirations and priming on well-being. Journal of cleaner production 18 (2010). Elsevier

${ }^{29}$ LA TRAGEDIA DE LOS COMUNES. Garrett Hardin

Este artículo fue publicado originalmente bajo el título "The Tragedy of Commons" en Science, v. 162 (1968), pp. 1243-1248. Traducción

de Horacio Bonfil Sánchez. Gaceta Ecológica, núm. 37, Instituto Nacional de Ecología, México, 1995. Http://www.ine.gob.mX/

${ }^{30}$ LA CARTA DEL URBANISMO EUROPEO BARCELONA 2013. INNOVACIÓN, CREATIVIDAD Y TERRITORIOS INTELIGENTES

Arantxa Rodríguez y Marisol Esteban. Universidad del País Vasco / Euskal Herriko Unibertsitatea.

${ }^{31} \mathrm{La}$ ciudad compacta y diversa frente a la conurbación difusa. Salvador Rueda. http://habitat.aq.upm.es/cs/p2/a009.html

${ }^{32} \mathrm{M}$. Kärrholm, K. Nylund, P. Prieto de la Fuente. Spatial resilience and urban planning: addressing the interdependence of urban retail areas. Cities 36. (2014). Elsevier

33 J. R. Fernandes, P. Chamusca. Urban policies, planning and retail resilience. Cities 36. (2014). Elsevier

$34 \mathrm{~J}$. Ahern. From fail-safe to safe-to-fail: sustainability and resilience in the new urban world. Landscape and urban planning 100. (2011)

${ }^{35}$ M. J. Collier, Z. Nedovic'-Budic', J. Aerts, S. Connop, D. Foley, K. Foley, D. Newport, S. Mcquaid, A. Slaev, P. Verburg. Transitioning to resilience and sustainability in urban communities. Cities 32. (2013)

${ }^{36}$ K.C. Desouza, T.H. Flanery. Designing, planning, and managing resilient cities: a conceptual framework. Cities 35 (2013) 89-99

${ }^{37}$ Y. Jabareen. Planning the resilient city: concepts and strategies for coping with climate change and environmental risk. Cities 31 (2013) 220-229. Elsevier

${ }^{38}$ W. Mcdonough, M. Braungart. Craddle to Craddle (2005)

39 Towards a circular economy: a zero waste programme for Europe. European commission Brussels, com (2014) 398 final. Communication from the commission to the European parliament, the council, the European economic and social committee and the committee of the regions

${ }^{40}$ DESING AFTER DECLINE. HOW AMERICA REBUILDS SHRINKING CITIES. Brent D. Ryan, URBAN DESIGN AND PUBLIC POLICY. MASSACHUSETTS INSTITUTE OF TECHNOLOGY.

${ }^{41}$ F. Gaja i Díaz. Urbanismo, ciudades y calentamiento global. Equívocos y sofismas (2010). Seminario-taller. "Ciudad y región urbana en la perspectiva de calentamiento global"

42 TOWARDS A CIRCULAR ECONOMY: A ZERO WASTE PROGRAMME FOR EUROPE.

EUROPEAN COMMISSION Brussels, 2.7.2014 COM (2014) 398 final

COMMUNICATION FROM THE COMMISSION TO THE EUROPEAN PARLIAMENT, THE COUNCIL, THE EUROPEAN ECONOMIC AND SOCIAL COMMITTEE AND THE COMMITTEE OF THE REGIONS

${ }^{43}$ K. Tidball, R. Stedman. Positive dependency and virtuous cycles: from resource dependence to resilience in urban social-ecological systems. Ecological economics 86. (2013).

${ }^{44}$ http://www.eib.org/infocentre/events/all/csi-europe.htm 
${ }^{45}$ http://orbenismo.es/category/derechos-y-deberes/d-conservacion/

${ }_{46}$ CAMBIO GLOBAL ESPAÑA 2020/50. SECTOR EDIFICACIÓN. 2010. La imprescindible reconversión del sector frente al reto de la sostenibilidad. PROGRAMA CAMBIO GLOBAL ESPAÑA 2020/50 DEL CENTRO COMPLUTENSE DE ESTUDIOS E INFORMACIÓN MEDIOAMBIENTAL DE LA FUNDACIÓN GENERAL DE LA UNIVERSIDAD COMPLUTENSE DE MADRID. 